\title{
Heavy Metals Status in Some Commercially Important Fishes of Meghna River Adjacent to Narsingdi District, Bangladesh: Health Risk Assessment
}

\author{
Md. Simul Bhuyan ${ }^{1, ~ *, ~ M u h a m m a d ~ A b u ~ B a k a r ², ~ A y s h a ~ A k h t a r ~}{ }^{1}$, Md. Shafiqul Islam ${ }^{1}$ \\ ${ }^{1}$ Institute of Marine Sciences and Fisheries, University of Chittagong, Chittagong, Bangladesh \\ ${ }^{2}$ Bangladesh Council of Scientific and Industrial Research, Chittagong, Bangladesh
}

Email address:

simulbhuyan@gmail.com (Md. S. Bhuyan)

${ }^{*}$ Corresponding author

\section{To cite this article:}

Md. Simul Bhuyan, Muhammad Abu Bakar, Md. Shafiqul Islam, Aysha Akhtar. Heavy Metals Status in Some Commercially Important Fishes of Meghna River Adjacent to Narsingdi District, Bangladesh: Health Risk Assessment. American Journal of Life Sciences. Vol. 4, No. 2, 2016, pp. 60-70. doi: 10.11648/j.ajls.20160402.17

Received: April 27, 2016; Accepted: May 6, 2016; Published: May 30, 2016

\begin{abstract}
The present study was carried out to determine the heavy metals $(\mathrm{Cd}, \mathrm{Pb}, \mathrm{Zn}, \mathrm{Al}, \mathrm{Cu}, \mathrm{Ni}, \mathrm{Fe}, \mathrm{Mn}, \mathrm{Cr}, \mathrm{Co})$ in the muscles of 32 fish species for 3 seasons in Meghna River. The heavy metals were analyzed by Atomic Absorption Spectrophotometer. The estimated concentrations of all metals in the present study were lower than the limits permitted by FAO, WHO, EU, United States Food and Drug Administration (USFDA), US Environment Protection Agency (US/EPA) and England Guidelines except the concentrations of $\mathrm{Pb} \& \mathrm{Zn}$ that were found above the allowable ranges in different fishes namely Amblypharyngnodon mola. Colisa lalia, Tetraodon cutcutia, Barbodes sarana, Labeo calbasu, Puntius sarena, Ompok pabda, Aila coila, Mastacembelus armatus, Glossogobius giuris, Nandus nandu, Tenualosa ilisha, Lepidocephalichthys guntea, Xenentodon cancila, Stinging catfish. Multivariate statistical analysis such as principal component analysis and correlation matrix showed significant anthropogenic intrusions of $\mathrm{Zn}, \mathrm{Al}, \mathrm{Cd}, \mathrm{Pb}, \mathrm{Cu}, \mathrm{Ni}, \mathrm{Fe}, \mathrm{Mn}, \mathrm{Cr}, \mathrm{Co}$ in fishes. There was significant positive correlation between $\mathrm{Cd}$ vs $\mathrm{Co}(0.733)$, moderate positive correlation between $\mathrm{Fe}$ vs $\mathrm{Al}(0.568)$, $\mathrm{Ni}$ vs $\mathrm{Co}$ (0.482), Mn vs Co (0.395) which indicate that their common origin especially from industrial effluents, municipal wastes and agricultural inputs.
\end{abstract}

Keywords: Heavy Metal, Multivariate Analysis, Fish, Atomic Absorption Spectrophotometer, Meghna River

\section{Introduction}

Industrialization is a term associated with socio-economic activities (Richard 2005; Jaillon and Poon 2009, Thanoon et al. 2003) that alter the society infrastructure (Abdullah et al. 2009) through the huge production (Abdullah et al. 2009; Thanoon et al. 2003). Most of these industries discharge untreated wastes that contribute heavy metal hugely. Industrial and agricultural as well as natural activities are the leading responsible sources of metal contamination in aquatic environment (Wilson and Pyatt 2007; Khan et al. 2008; Tarra-Wahlberg et al. 2001; Akif et al. 2002; Jordao et al. 2002; Sekabira et al. 2010; Zhang et al. 2011; Bai et al. 2011; Grigoratos et al. 2014; Martin et al. 2015). Heavy metal pollution is a great concern because of their long persistence, bioaccumulation and biomagnifications in the food chain (Rahman et al. 2013; Sharma et al. 2007; Sankar et al. 2006; Papagiannis et al. 2004; Zhou et al. 2004; Sun et al. 2001; Zhou 1995) ultimately poses toxicity both in human and aquatic animals (Islam et al. 2015a; Ahmed et al. 2015a, b; Fang et al. 2014; Alhashemi et al. 2012; Pan and Wang 2012; Yi et al. 2011; Vieira et al. 2011; Forti et al. 2011; Banerjee et al. 2011; Tuzen 2009; Sanchez-Chardi et al. 2007; McCluggage 1991; WHO 1995). Most of the literature conducted on bioaccumulation and toxicity of heavy metal (Rainbow et al. 2000; Shuhaimi-Othmana and Pascoe 2007) as the heavy metal pollution is global concern (Islam et al. 2014). This increasing pattern of heavy metals have adverse health effects for invertebrates, fish, and humans (Yi et al. 2011; Islam et al. 2014; Martin et al. 2015; Islam et al. 
2015b,d; Ahmed et al. 2015c). Recently, in many developing countries like Bangladesh are continuously being contaminated with heavy metal (Islam et al. 2015c) due to disposal of untreated effluents from different industries, domestic wastes and agrochemicals in the open water bodies and rivers deteriorating water quality (Khadse et al. 2008; Venugopal et al. 2009; Islam et al. 2015a,c). Heavy metal in aquatic ecosystems measured by monitoring concentrations in water, sediments and biota (Camusso et al. 1995) but considerable amount was found in fishes (Rashed 2001) and sediments (Namminga and Wilhm 1976). Several studies have been conducted in rivers and lakes giving special preference to environment during the last decade (Özmen et al. 2004; Begüm et al. 2005; Fernandes et al. 2008; Öztürk et al. 2008; Pote et al. 2008 and Praveena et al. 2008). Meghna River near Narsingdi is highly polluted with industrial and domestic sewage that contribute huge amount of $\mathrm{Zn}, \mathrm{Al}, \mathrm{Cd}$, $\mathrm{Pb}, \mathrm{Cu}, \mathrm{Ni}, \mathrm{Fe}, \mathrm{Mn}, \mathrm{Cr}, \mathrm{Co}$. But unfortunately, there was no scientific research on heavy metal pollution in the concerned area was found so far which compelled us to conduct this research with aim (1) to determine the heavy metal concentrations in fish and (2) to compare the concentrations of heavy metal in different seasons.

\section{Materials and Methods}

\subsection{Sampling Sites}

Fish samples were collected from two points (Effluent discharge area and far from the discharge area) of Meghna River near Narsingdi District $\left(23^{\circ} 55^{\prime} 28.52^{\prime \prime} \mathrm{N}\right.$ and $\left.90^{\circ} 45^{\prime} 12.06 " \mathrm{E}\right)$. Sampling procedures were performed in three phases: firstly, September, 2015 (Rainy season); secondly, January, 2016 (Winter season) and thirdly, March, 2016 (Pre-monsoon).

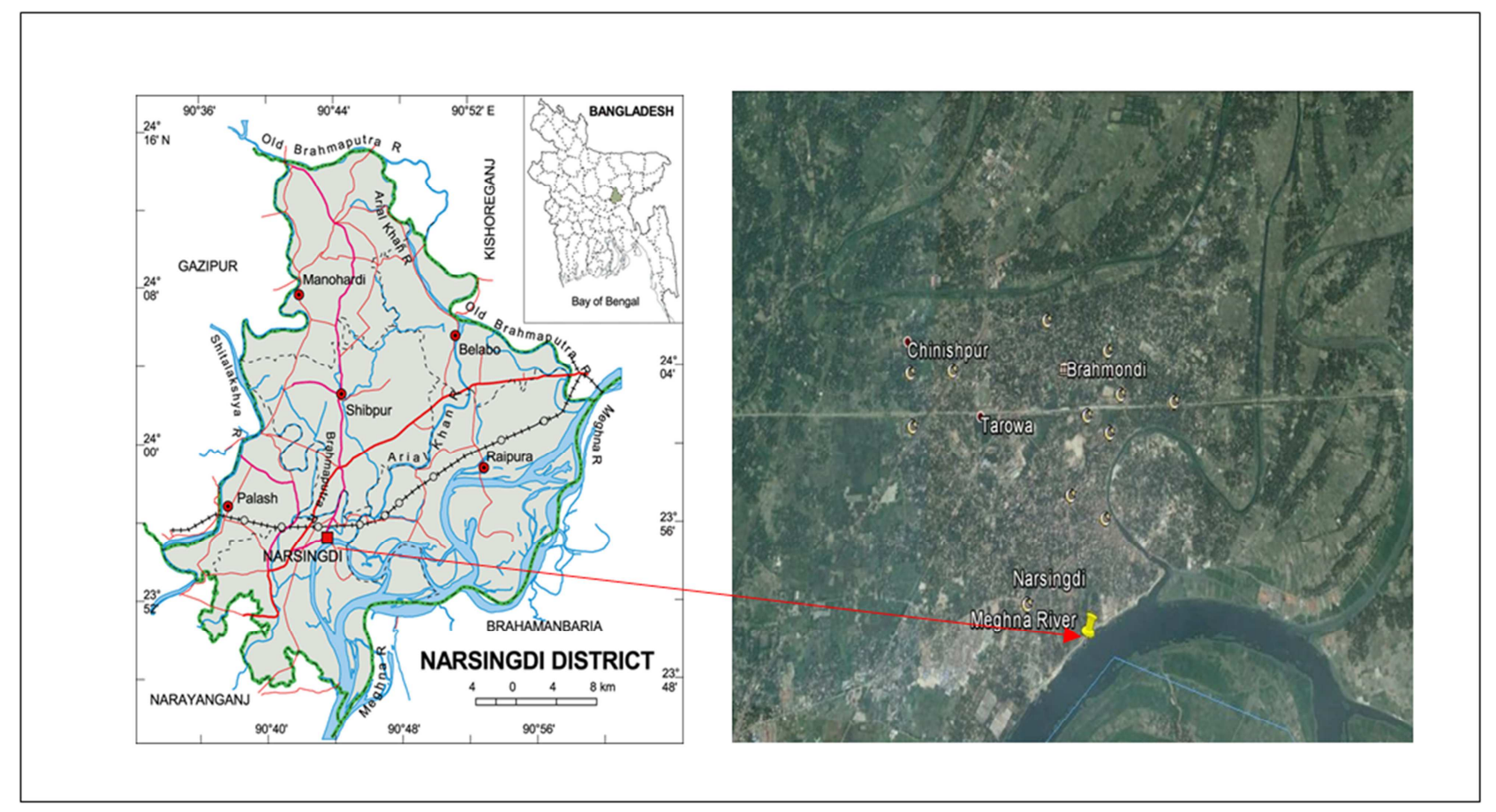

Figure 1. Map showing sampling station of Meghna River.

\subsection{Sample Collection and Preservation}

A total of 32 fish species were collected from fishermen for individual season and then identified according to Rahman et al. (2009), Roy et al. (2007), Quddus et al. (1988), Quddus and Shafi (1983). After collection the fish were placed immediately in poly-ethylene bags and then kept into isolated container of polystyrene icebox. Finally, the samples were transferred to the Bangladesh Council of Scientific and Industrial Research (BCSIR) in ice box (Irwandi and Farida 2009; Ismail and Saleh 2012) where the fish were first washed with deionized water sealed in poly-ethylene bags subsequently kept in a freezer at $-20^{\circ} \mathrm{C}$ until analysis (Elnabris et al. 2012).

\subsection{Heavy Metal Determination}

The heavy metal contents were determined by AAS using standard analytical procedure. Sample collection is important stage for metal analysis. Samples were generally carefully handled to avoid contamination. Glassware was properly cleaned, and the reagents were of analytical grade. Distilled water was used throughout the study. Reagents blank determinations were used to correct the instrument readings. The techniques for samples preparation, standard Preparation, analysis for metal analyses have been briefly described below.

\subsection{Sample Preparation (Dry Ashing Method)}

This procedure was also used for destruction of organic 
matter. Precaution was to be taken to avoid losses by volatilization of elements. At first samples were homogenized. Then the samples were weighed accurately a suitable quantity (10 to $20 \mathrm{~g}$ ) of the homogenized samples in a tared silica dish. After that the samples were dried at $100^{\circ} \mathrm{C}$ in a laboratory oven. These dishes were then placed in the muffle furnace at ambient temperature and slowly raised temperature to $450^{\circ} \mathrm{C}$ at a rate of no more than $50^{\circ} \mathrm{C} / \mathrm{h}$. The samples were ignited in a Muffle furnace at $450^{\circ} \mathrm{C}$ for at least 8 hrs. After ashing was completed and cool, then the dishes were removed from furnace. Then the ashes were dissolved in diluted nitric acid (Afthan et al. 2000). The solutions were returned to a hot plate and continued heating, adding additional acid as necessary until digestion was completed. Then the samples were filtrated into a $100 \mathrm{ml}$ volumetric flask using Whatman No. 44 filter paper and washed the residue. Each sample solution was made up to the mark with distilled water.

\subsection{Standard Preparation}

Every metal standard solution was prepared for calibration the instrument for each element being determined on the same day as the analyses were performed due to possible deterioration of standard with time. All samples were prepared from chemicals of analytical grade with distilled water. $1 \mathrm{gm}$ of metal Cadmium, Copper, Lead, Nickel were dissolved in $\mathrm{HNO}_{3}$ solution; $1 \mathrm{~g}$ of Cobalt, Iron, Manganese, Zinc, Aluminum were dissolved in $\mathrm{HCl}$ solution; $2.8289 \mathrm{~g} \mathrm{~K}_{2} \mathrm{Cr}_{2} \mathrm{O}_{7}$ (=1 $\mathrm{g}$ Chromium) was dissolved in water and made up to 1 liter in volumetric flask with distilled water, thus stock solution of $1000 \mathrm{mg} / \mathrm{l}$ of $\mathrm{Cd}, \mathrm{Cu}$, $\mathrm{Pb}, \mathrm{Ni}, \mathrm{Co}, \mathrm{Fe}, \mathrm{Mn}, \mathrm{Zn}, \mathrm{Al}$ and $\mathrm{Cr}$ were prepared. (Cantle, J. E. 1982). Then $100 \mathrm{ml}$ of $0.1,0.25,0.5,0.75,1.0$ and 2.0 $\mathrm{mg} / \mathrm{l}$ of working standards of each metal except iron were prepared from these stock using micropipettes in $5 \mathrm{ml}$ of $2 \mathrm{~N}$ nitric acid. $100 \mathrm{ml}$ of 2.0, 2.5, 5.0, 10.0 and $20.0 \mathrm{mg} / \mathrm{l}$ of working standards of iron metal were prepared from iron stock solution. Reagent blank was prepared in the same manners of sample preparation without sample to avoid reagents contamination.

\subsection{Analysis of Sample}

Finally, the atomic absorption instrument was set up carefully. At the meantime, flame condition and absorbance were optimized for the analyte. Then blanks (deionized water), standards, sample blank and samples were aspirated into the flame in AAS (Model- iCE 3300, Thermo Scientific, Designed in UK, Made by China)). The calibration curves were found for concentration vs. absorbance. Data were statistically analyzed using fitting of straight line by least square method. For more accuracy, a blank reading was also taken and necessary corrections were made during the calculation of various elements concentration.

\subsection{Statistical Analysis}

One Way Analysis of Variance (ANOVA) was done to show the variations in concentration of heavy metal in terms of seasons and fish. GGraph was used for graphical presentation of heavy metal against seasons. According to Dreher (2003), Principal Component Analysis (PCA) was performed on the original data set (without any weighting or standardization). Pearson's product moment correlation matrix was done to identify the relation among metals to make the result validate obtained from multivariate analysis.

\section{Results}

A total 32 fish species were analyzed for heavy metals ( $\mathrm{Zn}, \mathrm{Al}, \mathrm{Cd}, \mathrm{Pb}, \mathrm{Cu}, \mathrm{Ni}, \mathrm{Fe}, \mathrm{Mn}, \mathrm{Cr}, \mathrm{Co}$ ) detection. In rainy season, the highest concentration of $\mathrm{Zn}$ was recorded in Amblypharyngnodon mola $(42.45 \mathrm{mg} / \mathrm{kg})$ and the lowest was found in Ctenopharyngodon idella $(10.27 \mathrm{mg} / \mathrm{kg}$ ) (Table 1). The maximum concentration of $\mathrm{Al}$ recorded in Gudusiachapra $(117.55 \mathrm{mg} / \mathrm{kg})$ and minimum was found in Ctenopharyngodon idella $(1.85 \mathrm{mg} / \mathrm{kg})$ (Table 1). The highest concentration of $\mathrm{Mn}$ was recorded in Tetraodon cutcutia $(19.07 \mathrm{mg} / \mathrm{kg})$ and lowest was found in Ctenopharyngodon idella $(0.96 \mathrm{mg} / \mathrm{kg})$ (Table 1). The highest concentration of $\mathrm{Cd}$ in Ompok pabda $(0.12 \mathrm{mg} / \mathrm{kg}) ; \mathrm{Pb}$ in Colisa lalia (5.87); $\mathrm{Cu}$ in Barbodes sarana $(32.44 \mathrm{mg} / \mathrm{kg}) ; \mathrm{Ni}$ in Amblypharyngnodon mola $(0.76 \mathrm{mg} / \mathrm{kg}) ; \mathrm{Cr}$ in Anabus testudineus $(1.75 \mathrm{mg} / \mathrm{kg})$ and $\mathrm{Co}$ in Mystus bleekeri $(0.43 \mathrm{mg} / \mathrm{kg})$ whereas the lowest concentration for these metals were below detection limit (Table 1).

During winter season, the highest value of $\mathrm{Zn}$ was recorded in Amblypharyngnodon mola $(44.48 \mathrm{mg} / \mathrm{kg})$ and the lowest value was found in Johnius coitor $(9.6 \mathrm{mg} / \mathrm{kg}$ ) (Table 2). The highest concentration of Al was detected in Labeo rohita $(106.70 \mathrm{mg} / \mathrm{kg}$ ) and the lowest value was documented in Nandus nandus $(3.24 \mathrm{mg} / \mathrm{kg})$. The maximum value of $\mathrm{Cu}$ was measured in Glossogobius giuris $(8.19 \mathrm{mg} / \mathrm{kg})$ and the minimum value was recorded in Macrognathus aculeatus $(0.20 \mathrm{mg} / \mathrm{kg}$ ) (Table 2). The maximum amount of $\mathrm{Fe}$ was detected in Mystus bleekeri $(93.16 \mathrm{mg} / \mathrm{kg})$ and the lowest amount was found in Ctenopharyngodon idella $(9.86 \mathrm{mg} / \mathrm{kg})$. The highest concentration of Mn was found in Colisa lalia $(19.87 \mathrm{mg} / \mathrm{kg})$ and the lowest concentration was measured in Mastacembelus armatus $(1.65 \mathrm{mg} / \mathrm{kg})$. Moreover, the maximum amount was recorded for Cd in Ompok pabda $(0.21 \mathrm{mg} / \mathrm{kg}) ; \mathrm{Pb}$ in Colisa lalia $(6.75 \mathrm{mg} / \mathrm{kg}) ; \mathrm{Ni}$ in Amblypharyngnodon mola $(0.986 \mathrm{mg} / \mathrm{kg}) ; \mathrm{Cr}$ in Stinging catfish $(3.01 \mathrm{mg} / \mathrm{kg})$ and $\mathrm{Co}$ in Labeo rohita $(0.70 \mathrm{mg} / \mathrm{kg})$ while the lowest concentration of these metals were recorded below detection limit (Table 2).

In Pre-monsoon, the highest value of $\mathrm{Zn}$ was recorded in Amblypharyngnodon mola $(43.67 \mathrm{mg} / \mathrm{kg})$ and the lowest value was documented in Johnius coitor $(8.65 \mathrm{mg} / \mathrm{kg}$ ) (Table 3 ). The greatest amount of $\mathrm{Al}$ was measured in Labeo rohita $(104.87 \mathrm{mg} / \mathrm{kg})$ and the lowest amount was found in Ctenopharyngodon idella $(1.78 \mathrm{mg} / \mathrm{kg})$. The supreme concentration of $\mathrm{Cu}$ was detected in Barbodes sarana $(26.67 \mathrm{mg} / \mathrm{kg})$ and the lowest concentration was measured in Macrognathus aculeatus $(0.21 \mathrm{mg} / \mathrm{kg})$. The maximum value of Fe was recorded in Gudusiachapra $(77.66 \mathrm{mg} / \mathrm{kg})$ and the 
minimum value was recorded in Xenentodon cancila $(7.85 \mathrm{mg} / \mathrm{kg})$. The greatest amount of $\mathrm{Mn}$ was measured in Tetraodon cutcutia $(20.01 \mathrm{mg} / \mathrm{kg})$ and the lowest value was found in Ctenopharyngodon idella $(1.01 \mathrm{mg} / \mathrm{kg})$. The highest concentration was recorded for $\mathrm{Cd}$ in Ompok pabda $(0.23 \mathrm{mg} / \mathrm{kg}) ; \mathrm{Pb}$ in Colisa lalia $(6.85 \mathrm{mg} / \mathrm{kg}) ; \mathrm{Ni}$ in Amblypharyngnodon mola $(0.98 \mathrm{mg} / \mathrm{kg}) ; \mathrm{Cr}$ in Aila coila $(8.18 \mathrm{mg} / \mathrm{kg})$ and $\mathrm{Co}$ in Channa punctatus $(0.51 \mathrm{mg} / \mathrm{kg})$ whereas the lowest concentration for these metals was documented below detection limit (Table 3).

\subsection{Analysis of Variance (ANOVA) in Fish Species}

There were significant variations in the concentrations of Zinc, aluminium, lead, copper, nickel, iron, cobalt, manganese found across the various fish species as the significance level $(p<0.05)$. However, there were no significant variations $(\mathrm{p}>0.05)$ in cadmium and Chromium levels across the fish species. Moreover there was no prevalent variation $(p>0.05)$ in the metal concentration in terms of seasons except for lead and manganese $(p<0.05)$.

Table 1. Heavy metal concentrations ( $\mathrm{mg} / \mathrm{kg}$ ) in fish during Rainy season.

\begin{tabular}{|c|c|c|c|c|c|c|c|c|c|c|c|c|}
\hline $\begin{array}{l}\text { SI. } \\
\text { No. }\end{array}$ & Scientific Name & $\begin{array}{l}\text { Sample } \\
\text { ID }\end{array}$ & $\begin{array}{l}\mathrm{Zn} \\
(\mathrm{mg} / \mathrm{kg})\end{array}$ & $\begin{array}{l}\text { Al } \\
\text { (mg/kg) }\end{array}$ & $\begin{array}{l}\text { Cd } \\
(\mathrm{mg} / \mathrm{kg})\end{array}$ & $\begin{array}{l}\mathrm{Pb} \\
(\mathrm{mg} / \mathrm{kg})\end{array}$ & $\begin{array}{l}\mathrm{Cu} \\
(\mathrm{mg} / \mathrm{kg})\end{array}$ & $\begin{array}{l}\mathrm{Ni} \\
(\mathrm{mg} / \mathrm{kg})\end{array}$ & $\begin{array}{l}\text { Fe } \\
(\mathrm{mg} / \mathrm{kg})\end{array}$ & $\begin{array}{l}\text { Mn } \\
\text { (mg/kg) }\end{array}$ & $\begin{array}{l}\mathrm{Cr} \\
(\mathrm{mg} / \mathrm{kg})\end{array}$ & $\begin{array}{l}\text { Co } \\
\text { (mg/kg) }\end{array}$ \\
\hline 01 & Aila coila & FR-01 & 23.85 & 9.70 & BDL & $0 . \mathrm{O} 4$ & 0.19 & 0.35 & 18.62 & 3.45 & BDL & BDL \\
\hline 02 & Aorichthys aor & FR-02 & 19.54 & 6.20 & 0.027 & BDL & 0.61 & 0.192 & 18.61 & 2.02 & BDL & 0.24 \\
\hline 03 & Mastacembelus armatus & FR-03 & 15.38 & 2.10 & $\mathrm{BDL}$ & BDL & 1.41 & BDL & 24.34 & 1.76 & BDL & BDL \\
\hline 04 & Labeo bata & FR-04 & 15.67 & 12.99 & $\mathrm{BDL}$ & $\mathrm{BDL}$ & 0.93 & 0.08 & 19.62 & 3.56 & BDL & BDL \\
\hline 05 & Glossogobius giuris & FR-05 & 18.54 & 5.32 & BDL & BDL & 7.19 & 0.18 & 13.07 & 2.98 & BDL & BDL \\
\hline 06 & Nandus nandus & FR-06 & 19.70 & 3.51 & 0.015 & BDL & 1.02 & 0.138 & 17.23 & 4.8 & BDL & 0.27 \\
\hline 08 & Gudusiachapra & FR-08 & 17.7 & 117.55 & BDL & $\mathrm{BDL}$ & 1.01 & 0.22 & 76.21 & 14.21 & BDL & BDL \\
\hline 09 & Chitala chitala & FR-09 & 15.40 & 13.85 & $\mathrm{BDL}$ & 0.035 & 0.72 & $\mathrm{BDL}$ & 45.34 & 2.30 & 0.19 & BDL \\
\hline 10 & Colisa chuna & FR-10 & 14.52 & 12.55 & $\mathrm{BDL}$ & 0.09 & 1.40 & $\mathrm{BDL}$ & 35.76 & 3.75 & 0.78 & BDL \\
\hline 11 & Barbodes sarana & FR-11 & 24.41 & 45.52 & 0.099 & 0.47 & 32.44 & 0.193 & 39.97 & 4.46 & BDL & 0.28 \\
\hline 12 & Tenualosa ilisha & FR-12 & 11.31 & 3.86 & 0.092 & 0.67 & 1.21 & 0.084 & 27.54 & 2.39 & 0.05 & BDL \\
\hline 13 & Notopterus notopterus & FR-13 & 18.62 & 13.21 & $\mathrm{BDL}$ & BDL & 0.03 & 0.01 & 19.23 & 3.67 & $\mathrm{BDL}$ & BDL \\
\hline 14 & Tetraodon cutcutia & FR-14 & 34.21 & 33.01 & BDL & $\mathrm{BDL}$ & 0.99 & 0.31 & 39.91 & 19.07 & BDL & BDL \\
\hline 17 & Mystus cavasius & FR-17 & 15.82 & 27.41 & BDL & BDL & 0.84 & 0.272 & 35.74 & 1.50 & BDL & BDL \\
\hline 18 & Lepidocephalichthys guntea & FR-18 & 24.56 & 7.88 & $\mathrm{BDL}$ & 3.21 & 2.30 & 0.29 & 14.87 & 3.77 & BDL & 0.03 \\
\hline 19 & Puntius sarena & FR-19 & 29.65 & 44.28 & 0.035 & BDL & 0.71 & 0.210 & 66.71 & 9.44 & BDL & BDL \\
\hline 20 & Xenentodon cancila & FR-20 & 20.13 & 5.45 & BDL & 0.38 & 1.21 & 0.187 & 18.71 & 2.56 & BDL & 0.27 \\
\hline 21 & Clupisoma garua & FR-21 & 23.01 & 21.44 & $\mathrm{BDL}$ & $\mathrm{BDL}$ & 0.38 & 0.12 & 42.42 & 10.10 & BDL & BDL \\
\hline 22 & Anabus testudineus & FR-22 & 26.44 & 17.72 & $\mathrm{BDL}$ & BDL & 1.54 & 0.185 & 65.43 & 7.10 & 1.75 & BDL \\
\hline 23 & Amblypharyngnodon mola & FR-23 & 42.45 & 31.11 & $\mathrm{BDL}$ & BDL & 2.05 & 0.76 & 28.04 & 5.43 & BDL & BDL \\
\hline 24 & Ompok pabda & FR-24 & 39.50 & 4.5 & 0.121 & $\mathrm{BDL}$ & 0.64 & 0.362 & 11.16 & 1.92 & BDL & 0.41 \\
\hline 25 & Johnius coitor & FR-25 & 17.34 & 9.32 & BDL & BDL & 0.50 & 0.10 & 31.36 & 3.10 & 0.21 & 0.30 \\
\hline 26 & Labeo rohita & FR-26 & 28.40 & 103.13 & 0.013 & $\mathrm{BDL}$ & 1.98 & 0.150 & 64.56 & 9.76 & 0.21 & 0.37 \\
\hline 27 & Stinging catfish & FR-27 & 12.83 & 15.37 & 0.063 & 1.35 & 1.02 & $\mathrm{BDL}$ & 93.83 & 2.54 & 0.19 & 0.24 \\
\hline 28 & Channa striatus & FR-28 & 16.42 & 2.45 & $\mathrm{BDL}$ & BDL & 0.61 & 0.084 & 39.21 & 9.10 & 0.08 & 0.15 \\
\hline 29 & Channa punctatus & FR-29 & 15.59 & 6.10 & 0.011 & 0.040 & 1.27 & 0.12 & 147.77 & 9.55 & BDL & 0.29 \\
\hline 30 & Macrognathus aculeatus & FR-30 & 20.32 & 4.89 & BDL & BDL & 0.10 & 0.11 & 18.32 & 2.67 & BDL & $\mathrm{BDL}$ \\
\hline 31 & Mystus bleekeri & FR-31 & 28.15 & 10.90 & 0.039 & $\mathrm{BDL}$ & 1.41 & BDL & 78.35 & 2.77 & BDL & 0.43 \\
\hline
\end{tabular}

Table 2. Heavy metal concentrations ( $\mathrm{mg} / \mathrm{kg}$ ) in fish during Winter season.

\begin{tabular}{|c|c|c|c|c|c|c|c|c|c|c|c|c|}
\hline $\begin{array}{l}\text { Sl. } \\
\text { No. }\end{array}$ & Scientific Name & $\begin{array}{l}\text { Sample } \\
\text { ID }\end{array}$ & $\begin{array}{l}\mathrm{Zn} \\
(\mathrm{mg} / \mathrm{kg})\end{array}$ & $\begin{array}{l}\text { Al } \\
\text { (mg/kg) }\end{array}$ & $\begin{array}{l}\text { Cd } \\
(\mathrm{mg} / \mathrm{kg})\end{array}$ & $\begin{array}{l}\text { Pb } \\
(\mathrm{mg} / \mathrm{kg})\end{array}$ & $\begin{array}{l}\mathrm{Cu} \\
(\mathrm{mg} / \mathrm{kg})\end{array}$ & $\begin{array}{l}\mathrm{Ni} \\
(\mathrm{mg} / \mathrm{kg})\end{array}$ & $\begin{array}{l}\text { Fe } \\
(\mathrm{mg} / \mathrm{kg})\end{array}$ & $\begin{array}{l}\text { Mn } \\
(\mathrm{mg} / \mathrm{kg})\end{array}$ & $\begin{array}{l}\mathrm{Cr} \\
(\mathrm{mg} / \mathrm{kg})\end{array}$ & $\begin{array}{l}\text { Co } \\
\text { (mg/kg) }\end{array}$ \\
\hline 01 & Aila coila & FW-01 & 25.77 & 10.69 & BDL & 0.15 & 0.24 & 0.456 & 23.63 & 3.51 & BDL & BDL \\
\hline 02 & Aorichthys aor & FW-02 & 14.96 & 43.19 & BDL & BDL & 0.40 & 0.235 & 54.77 & 2.40 & 0.20 & $\mathrm{BDL}$ \\
\hline 03 & Mastacembelus armatus & FW-03 & 14.99 & 6.15 & BDL & BDL & 1.98 & 0.141 & 20.43 & 1.65 & BDL & BDL \\
\hline 04 & Labeo bata & FW-04 & 16.75 & 14.35 & BDL & BDL & 1.10 & 0.174 & 21.92 & 4.34 & BDL & BDL \\
\hline 05 & Glossogobius giuris & FW-05 & 19.63 & 5.52 & BDL & $\mathrm{BDL}$ & 8.19 & 0.179 & 15.05 & 3.22 & BDL & BDL \\
\hline 06 & Nandus nandus & FW-06 & 17.85 & 3.24 & $\mathrm{BDL}$ & BDL & 0.91 & 0.112 & 19.12 & 10.41 & $\mathrm{BDL}$ & BDL \\
\hline 08 & Gudusiachapra & FW-08 & 18.73 & 120.4 & BDL & BDL & 1.21 & 0.297 & 78.62 & 14.34 & BDL & $\mathrm{BDL}$ \\
\hline 09 & Chitala chitala & FW-09 & 11.47 & 5.90 & BDL & BDL & 0.45 & 0.093 & 13.50 & 2.65 & BDL & BDL \\
\hline 10 & Colisa chuna & FW-10 & 15.99 & 13.79 & BDL & 0.11 & 2.11 & $\mathrm{BDL}$ & 38.67 & 4.87 & 1.6 & BDL \\
\hline 11 & Barbodes sarana & FW-11 & 16.10 & 10.53 & 0.030 & BDL & 6.52 & 0.129 & 14.10 & 3.54 & BDL & BDL \\
\hline 12 & Tenualosa ilisha & FW-12 & 11.31 & 3.86 & 0.092 & 0.67 & 1.21 & 0.084 & 27.54 & 2.39 & 0.05 & BDL \\
\hline 13 & Notopterus notopterus & FW-13 & 19.47 & 13.50 & BDL & BDL & 0.33 & 0.130 & 23.07 & 4.91 & BDL & BDL \\
\hline 14 & Tetraodon cutcutia & FW-14 & 35.95 & 34.51 & BDL & BDL & 1.47 & 0.364 & 42.16 & 19.58 & BDL & $\mathrm{BDL}$ \\
\hline
\end{tabular}




\begin{tabular}{|c|c|c|c|c|c|c|c|c|c|c|c|c|}
\hline $\begin{array}{l}\text { SI. } \\
\text { No. }\end{array}$ & Scientific Name & $\begin{array}{l}\text { Sample } \\
\text { ID }\end{array}$ & $\begin{array}{l}\mathrm{Zn} \\
(\mathrm{mg} / \mathrm{kg})\end{array}$ & $\begin{array}{l}\begin{array}{l}\text { Al } \\
(\mathrm{mg} / \mathrm{kg})\end{array} \\
\end{array}$ & $\begin{array}{l}\begin{array}{l}\text { Cd } \\
(\mathrm{mg} / \mathrm{kg})\end{array} \\
\end{array}$ & $\begin{array}{l}\mathrm{Pb} \\
(\mathrm{mg} / \mathrm{kg})\end{array}$ & $\begin{array}{l}\mathrm{Cu} \\
(\mathrm{mg} / \mathrm{kg})\end{array}$ & $\begin{array}{l}\mathrm{Ni} \\
(\mathrm{mg} / \mathrm{kg})\end{array}$ & $\begin{array}{l}\mathrm{Fe} \\
(\mathrm{mg} / \mathrm{kg})\end{array}$ & $\begin{array}{l}\mathrm{Mn} \\
(\mathrm{mg} / \mathrm{kg})\end{array}$ & $\begin{array}{l}\mathrm{Cr} \\
(\mathrm{mg} / \mathrm{kg})\end{array}$ & $\begin{array}{l}\text { Co } \\
(\mathrm{mg} / \mathrm{kg})\end{array}$ \\
\hline 16 & Labeo calbasu & FW-16 & 31.21 & 43.87 & BDL & 1.91 & 1.76 & $\mathrm{BDL}$ & 20.01 & 4.79 & 1.12 & $\mathrm{BDL}$ \\
\hline 17 & Mystus cavasius & FW-17 & 17.21 & 27.69 & BDL & BDL & 1.02 & 0.31 & 36.74 & 1.81 & BDL & BDL \\
\hline 18 & $\begin{array}{l}\text { Lepidocephalichthys } \\
\text { guntea }\end{array}$ & FW-18 & 25.99 & 9.01 & BDL & 4.21 & 2.45 & 0.41 & 15.89 & 4.65 & BDL & 0.067 \\
\hline 19 & Puntius sarena & FW-19 & 39.65 & 12.28 & BDL & BDL & 1.23 & 0.596 & 33.96 & 15.99 & BDL & BDL \\
\hline 20 & Xenentodon cancila & FW-20 & 19.05 & 6.92 & BDL & BDL & 0.39 & 0.307 & 13.94 & 3.40 & BDL & BDL \\
\hline 21 & Clupisoma garua & FW-21 & 24.12 & 22.24 & BDL & $\mathrm{BDL}$ & 0.52 & 0.227 & 44.40 & 11.43 & BDL & BDL \\
\hline 22 & Anabus testudineus & FW-22 & 15.61 & 3.32 & BDL & BDL & 0.82 & 0.092 & 27.10 & 6.97 & 0.19 & BDL \\
\hline 23 & Amblypharyngnodon mola & FW-23 & 44.48 & 32.0 & BDL & BDL & 2.26 & 0.986 & 30.05 & 5.74 & BDL & BDL \\
\hline 24 & Ompok pabda & FW-24 & 39.98 & 5.01 & 0.21 & BDL & 0.69 & 0.38 & 12.01 & 2.05 & BDL & 0.51 \\
\hline 25 & Johnius coitor & FW-25 & 9.60 & 29.10 & BDL & BDL & 0.34 & 0.157 & 23.92 & 2.85 & 0.057 & BDL \\
\hline 26 & Labeo rohita & FW-26 & 29.40 & 106.70 & 0.04 & BDL & 2.01 & 0.17 & 66.98 & 11.30 & 0.57 & 0.70 \\
\hline 27 & Stinging catfish & FW-27 & 13.01 & 11.42 & BDL & 1.56 & 1.76 & 0.45 & 25.94 & 3.89 & 3.01 & BDL \\
\hline 28 & Channa striatus & FW-28 & 16.67 & 3.42 & BDL & BDL & 1.01 & 0.10 & 40.01 & 10.21 & 0.10 & 0.22 \\
\hline 29 & Channa punctatus & FW-29 & 12.40 & 3.22 & BDL & BDL & 0.32 & 0.087 & 12.93 & 8.67 & BDL & BDL \\
\hline 30 & Macrognathus aculeatus & FW-30 & 22.55 & 5.57 & BDL & BDL & 0.20 & 0.243 & 19.38 & 3.58 & BDL & BDL \\
\hline 31 & Mystus bleekeri & FW-31 & 25.47 & 93.65 & BDL & BDL & 0.41 & 0.766 & 93.16 & 7.80 & BDL & BDL \\
\hline 32 & Silonia silondia & FW-32 & 23.24 & 4.21 & BDL & BDL & 0.54 & 0.18 & 29.92 & 1.86 & BDL & BDL \\
\hline
\end{tabular}

Table 3. Heavy metal concentrations ( $\mathrm{mg} / \mathrm{kg}$ ) in fish during Pre-monsoon season.

\begin{tabular}{|c|c|c|c|c|c|c|c|c|c|c|c|c|}
\hline $\begin{array}{l}\text { Sl. } \\
\text { No. }\end{array}$ & Scientific Name & $\begin{array}{l}\text { Sample } \\
\text { ID }\end{array}$ & $\begin{array}{l}\mathrm{Zn} \\
(\mathrm{mg} / \mathrm{kg})\end{array}$ & $\begin{array}{l}\text { Al } \\
(\mathrm{mg} / \mathrm{kg})\end{array}$ & $\begin{array}{l}\text { Cd } \\
(\mathrm{mg} / \mathrm{kg})\end{array}$ & $\begin{array}{l}\mathrm{Pb} \\
(\mathrm{mg} / \mathrm{kg})\end{array}$ & $\begin{array}{l}\mathrm{Cu} \\
(\mathrm{mg} / \mathrm{kg})\end{array}$ & $\begin{array}{l}\mathrm{Ni} \\
(\mathrm{mg} / \mathrm{kg})\end{array}$ & $\begin{array}{l}\mathrm{Fe} \\
(\mathrm{mg} / \mathrm{kg})\end{array}$ & $\begin{array}{l}\text { Mn } \\
(\mathrm{mg} / \mathrm{kg})\end{array}$ & $\begin{array}{l}\mathrm{Cr} \\
(\mathrm{mg} / \mathrm{kg})\end{array}$ & $\begin{array}{l}\begin{array}{l}\text { Co } \\
(\mathrm{mg} / \mathrm{kg})\end{array} \\
\end{array}$ \\
\hline 01 & Aila coila & FPM-01 & 26.84 & 9.43 & $\mathrm{BDL}$ & 3.28 & 1.41 & 0.182 & 17.93 & 3.65 & 8.18 & BDL \\
\hline 02 & Aorichthys aor & FPM-02 & 14.96 & 43.19 & BDL & BDL & 0.40 & 0.235 & 54.77 & 2.40 & 0.20 & BDL \\
\hline 03 & Mastacembelus armatus & FPM-03 & 11.59 & 5.10 & BDL & 1.34 & 1.26 & 0.04 & 12.36 & 2.86 & 1.94 & 0.17 \\
\hline 04 & Labeo bata & FPM-04 & 15.00 & 14.21 & BDL & BDL & 1.08 & 0.16 & 20.41 & 4.07 & BDL & BDL \\
\hline 05 & Glossogobius giuris & FPM-05 & 18.90 & 17.05 & BDL & 2.70 & 1.30 & 0.121 & 13.50 & 4.26 & BDL & 0.12 \\
\hline 06 & Nandus nandus & FPM-06 & 14.61 & 5.67 & BDL & 0.86 & 1.26 & 0.045 & 11.40 & 6.27 & 0.19 & 0.05 \\
\hline 07 & Colisa lalia & FPM-07 & 35.49 & 32.48 & BDL & 6.85 & 3.03 & $\mathrm{BDL}$ & 38.5 & 19.67 & BDL & $\mathrm{BDL}$ \\
\hline 08 & Gudusiachapra & FPM-08 & 18.01 & 119.3 & BDL & BDL & 1.06 & 0.28 & 77.66 & 14.84 & BDL & BDL \\
\hline 09 & Chitala chitala & FPM-09 & 10.98 & 5.72 & BDL & BDL & 0.46 & 0.08 & 12.78 & 2.45 & BDL & BDL \\
\hline 10 & Colisa chuna & FPM-10 & 15.77 & 12.98 & BDL & 0.12 & 1.56 & $\mathrm{BDL}$ & 36.21 & 3.90 & 0.40 & BDL \\
\hline 11 & Barbodes sarana & FPM-11 & 33.69 & 35.15 & $\mathrm{BDL}$ & 6.05 & 26.67 & 0.366 & 18.56 & 5.96 & $\mathrm{BDL}$ & BDL \\
\hline 12 & Tenualosa ilisha & FPM-12 & 11.01 & 3.91 & 0.07 & 0.59 & 1.11 & 0.01 & 27.03 & 2.21 & 0.07 & BDL \\
\hline 13 & Notopterus notopterus & FPM-13 & 19.15 & 9.00 & BDL & 2.54 & 0.78 & 0.182 & 19.08 & 6.22 & BDL & BDL \\
\hline 14 & Tetraodon cutcutia & FPM-14 & 34.21 & 34.01 & BDL & BDL & 1.40 & 0.31 & 41.87 & 20.01 & BDL & BDL \\
\hline 15 & Ctenopharyngodon idella & FPM-15 & 10.69 & 1.78 & BDL & BDL & 0.82 & 0.11 & 9.75 & 1.01 & 0.15 & BDL \\
\hline 16 & Labeo calbasu & FPM-16 & 30.80 & 43.57 & BDL & 1.89 & 1.65 & BDL & 19.84 & 4.50 & 1.04 & BDL \\
\hline 17 & Mystus cavasius & FPM-17 & 17.56 & 28.21 & BDL & BDL & 1.00 & 0.21 & 35.76 & 1.75 & BDL & BDL \\
\hline 18 & Lepidocephalichthys guntea & FPM-18 & 25.64 & 8.05 & BDL & 3.88 & 2.38 & 0.352 & 15.96 & 4.09 & BDL & 0.077 \\
\hline 19 & Puntius sarena & FPM-19 & 30.68 & 20.06 & BDL & 2.12 & 1.59 & 0.176 & 19.90 & 8.52 & BDL & BDL \\
\hline 20 & Xenentodon cancila & FPM-20 & 28.87 & 4.66 & BDL & 2.71 & 1.02 & 0.099 & 7.85 & 2.01 & 2.17 & 0.15 \\
\hline 21 & Clupisoma garua & FPM-21 & 24.32 & 20.22 & BDL & BDL & 0.41 & 0.21 & 41.65 & 10.98 & BDL & BDL \\
\hline 22 & Anabus testudineus & FPM-22 & 15.74 & 32.50 & BDL & 3.09 & 0.985 & 0.135 & 32.71 & 4.69 & 2.29 & 0.28 \\
\hline 23 & Amblypharyngnodon mola & FPM-23 & 43.67 & 31.55 & BDL & BDL & 2.43 & 0.98 & 30.10 & 4.32 & BDL & BDL \\
\hline 24 & Ompok pabda & FPM-24 & 38.45 & 4.50 & 0.23 & BDL & 0.63 & 0.28 & 11.47 & 2.10 & BDL & 0.42 \\
\hline 25 & Johnius coitor & FPM-25 & 8.65 & 27.70 & BDL & BDL & 0.32 & 0.16 & 22.47 & 2.67 & 0.04 & BDL \\
\hline 26 & Labeo rohita & FPM-26 & 29.20 & 104.87 & 0.03 & BDL & 1.99 & 0.17 & 66.22 & 9.88 & 0.31 & 0.45 \\
\hline 27 & Stinging catfish & FPM-27 & 10.84 & 10.80 & BDL & 1.66 & 1.22 & 0.073 & 24.84 & 2.79 & 1.97 & BDL \\
\hline 28 & Channa striatus & FPM-28 & 15.76 & 3.21 & $\mathrm{BDL}$ & BDL & 1.12 & 0.08 & 39.31 & 10.13 & 0.09 & 0.04 \\
\hline 29 & Channa punctatus & FPM-29 & 16.70 & 66.50 & BDL & 5.86 & 0.94 & BDL & 54.34 & 8.96 & 3.62 & 0.51 \\
\hline 30 & Macrognathus aculeatus & FPM-30 & 21.45 & 5.07 & BDL & BDL & 0.21 & 0.21 & 18.32 & 3.01 & BDL & BDL \\
\hline 31 & Mystus bleekeri & FPM-31 & 26.54 & 69.53 & $\mathrm{BDL}$ & 3.00 & 2.19 & $\mathrm{BDL}$ & 37.62 & 3.71 & 1.23 & BDL \\
\hline 32 & Silonia silondia & FPM-32 & 22.53 & 4.01 & BDL & BDL & 0.32 & 0.173 & 29.13 & 1.74 & BDL & BDL \\
\hline
\end{tabular}

FR - Fish Rainy Season

FW - Fish Winter Season

FPM- Fish Pre-monsoon

BDL - Below Detection Limit

\subsection{Correlation Matrix}

In aquatic environment, the inter relationship among metals in fishes provide significant information sources and pathways of variables. The result of correlations between heavy metals acquiesced with the results obtained by PCA and $\mathrm{CA}$ that confirm some new associations between parameters. There was significant positive correlation between $\mathrm{Cd}$ vs Co (0.733), moderate positive correlation 
between $\mathrm{Fe}$ vs $\mathrm{Al}$ (0.568), Ni vs Co (0.482), Mn vs Co (0.395) and weak correlation exist between $\mathrm{Cr}$ vs Co (0.351), $\mathrm{Pb}$ vs $\mathrm{Cr}(0.283)$. The strong and moderate correlation indicates their sources of origin are similar especially from industrial effluents, municipal wastes and agricultural inputs. On the other hand, weak negative correlations were found between $\mathrm{Cd}$ vs. $\mathrm{Zn}$ (-0.241), Pb vs. Ni (-.155), Cd vs. Fe ($0.147)$ in river fish.

Table 4. Correlation matrix of heavy metals in fish.

\begin{tabular}{|c|c|c|c|c|c|c|c|c|c|c|c|}
\hline & & $\mathrm{Zn}$ & Al & Cd & $\mathbf{P b}$ & $\mathrm{Cu}$ & $\mathrm{Ni}$ & $\mathrm{Fe}$ & Mn & $\mathrm{Cr}$ & Co \\
\hline \multirow{10}{*}{ Correlation } & $\mathrm{Zn}$ & 1.000 & & & & & & & & & \\
\hline & $\mathrm{Al}$ & .217 & 1.000 & & & & & & & & \\
\hline & $\mathrm{Cd}$ & -.241 & -.029 & 1.000 & & & & & & & \\
\hline & $\mathrm{Pb}$ & .247 & .071 & -.054 & 1.000 & & & & & & \\
\hline & $\mathrm{Cu}$ & .165 & .098 & -.039 & .261 & 1.000 & & & & & \\
\hline & $\mathrm{Ni}$ & .181 & .091 & .721 & -.155 & .013 & 1.000 & & & & \\
\hline & $\mathrm{Fe}$ & .070 & .568 & -.147 & -.087 & -.029 & -.075 & 1.000 & & & \\
\hline & $\mathrm{Mn}$ & .187 & .356 & .559 & .191 & -.010 & .446 & .253 & 1.000 & & \\
\hline & $\mathrm{Cr}$ & -.125 & .017 & .400 & .283 & -.059 & .215 & -.100 & .157 & 1.000 & \\
\hline & $\mathrm{Co}$ & -.054 & .119 & .733 & -.027 & .012 & .482 & .066 & .395 & .351 & 1.000 \\
\hline
\end{tabular}

\subsection{Principal Component Analysis}

The extraction method was used to find out the principal components in PCA analysis that was Eigen values. The components were taken as principal components whose Eigen values was greater than 0.6 were taken into account. 6 PCs were extracted by using correlation matrix which reflects the processes influencing the heavy metals composition having $88.16 \%$ of total sample variance (Table 4 ). The total variance of the PCs were $29.0 \%, 18.81 \%$ and $14.48 \%$,
$10.91 \%, 8.74 \%, 6.22 \%$ for PC 1, PC 2, PC 3, PC 4, PC 5, PC 6 respectively. PC 1 is strongly correlated with $\mathrm{Cd}, \mathrm{Co}, \mathrm{Ni}$, $\mathrm{Mn}$ and PC 2 with $\mathrm{Al}, \mathrm{Fe}, \mathrm{Zn}$. Moreover, there was strong correlation of $\mathrm{PC} 3$ with $\mathrm{Pb}, \mathrm{PC} 5$ with $\mathrm{Cu}$ and there was no strong correlation for PC 4 and PC 6 . The source of PC 1 and PC 2 can be considered as mixed source from anthropogenic inputs particularly from industrial effluents and agricultural activities in the study area. Whereas PC 3, PC 4, PC 5 and PC 6 can be considered as different source from both lithogenic and anthropogenic inputs.

Table 5. Component matrix of six factors model with strong to moderate loadings in fish.

\begin{tabular}{|c|c|c|c|c|c|c|}
\hline \multirow{2}{*}{ Parameters } & \multicolumn{6}{|c|}{ Component } \\
\hline & PC 1 & PC 2 & PC 3 & PC 4 & PC 5 & PC 6 \\
\hline $\mathrm{Cd}$ & .910 & -.315 & & & .101 & -.141 \\
\hline Co & .804 & & & & .178 & .121 \\
\hline $\mathrm{Ni}$ & .778 & & & -.453 & & .165 \\
\hline Mn & .717 & .381 & & & -.172 & -.469 \\
\hline $\mathrm{Al}$ & .234 & .784 & -.230 & .185 & & .162 \\
\hline $\mathrm{Fe}$ & & .697 & -.494 & .280 & .120 & \\
\hline $\mathrm{Zn}$ & & .567 & .352 & -.510 & -.418 & .263 \\
\hline $\mathrm{Pb}$ & & .289 & .793 & .345 & -.146 & -.250 \\
\hline $\mathrm{Cr}$ & .494 & -.201 & .335 & .578 & -.135 & .423 \\
\hline $\mathrm{Cu}$ & & .277 & .529 & -.236 & .749 & \\
\hline Eigen value & 2.9 & 1.88 & 1.45 & 1.10 & 0.87 & 0.62 \\
\hline$\%$ Total variance & 29.0 & 18.81 & 14.48 & 10.91 & 8.74 & 6.22 \\
\hline Cumulative \% & 29.0 & 47.81 & 62.29 & 73.20 & 81.94 & 88.16 \\
\hline
\end{tabular}

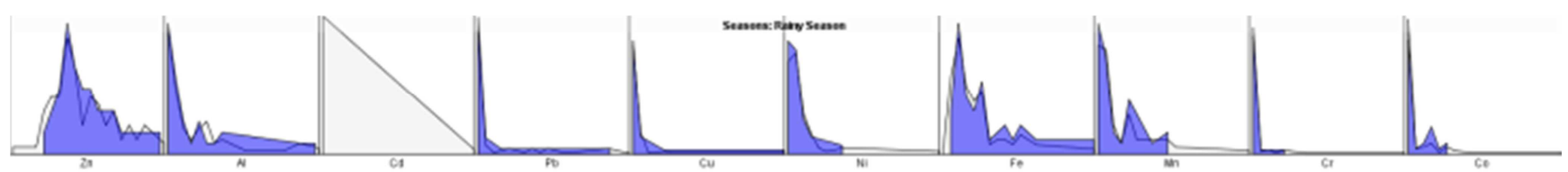

Figure 2. Concentration of different heavy metals in fishes during Rainy season.

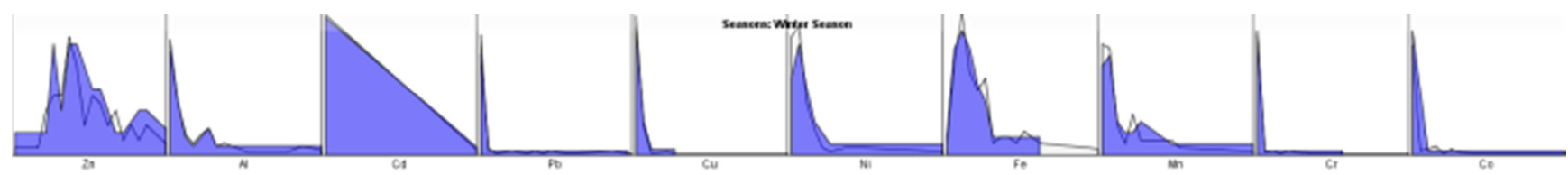

Figure 3. Concentration of different heavy metals in fishes during Winter season. 


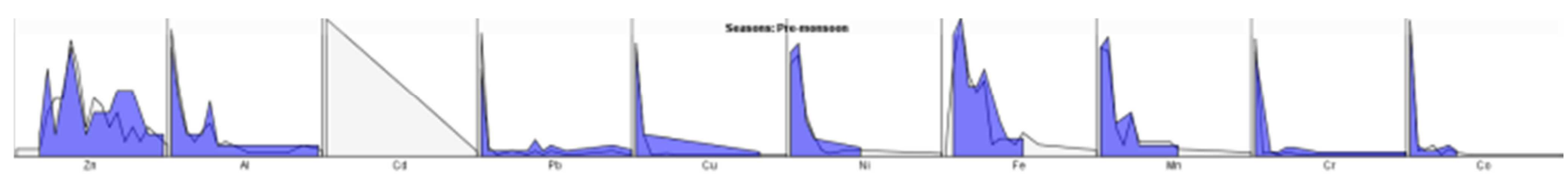

Figure 4. Concentration of different heavy metals in fishes during Pre-monsoon.

\section{Discussion}

The concentration of toxic metals in 32 fish species collected from Meghna River were found to be below the WHO permissible concentrations given for seafood (WHO 1972, 1987). Zinc is an important element in human diet but lower concentration can cause serious threat to human health (Agency for Toxic Substances and Disease Registry 2004). In the recent study the concentrations of $\mathrm{Zn}$ varied between $(8.65-44.48) \mathrm{mg} / \mathrm{kg}$. The concentration of $\mathrm{Zn}$ was lower than FAO maximum guideline of $30 \mathrm{mg} / \mathrm{kg}$ (FAO/WHO 1989; FAO 1983) except in Amblypharyngnodon mola. Colisa lalia, Tetraodon cutcutia, Barbodes sarana, Labeo calbasu, Puntius sarena, Ompok pabda for 3 consecutive seasons. The concentrations up to $40 \mathrm{mg} / \mathrm{kg}$ for $\mathrm{Zn}$ may induce toxicity, characterized by symptoms of irritability, muscular stiffness and pain, loss of appetite, and nausea (NAS-NRC 1974) though the toxicity of $\mathrm{Zn}$ rare.

In the present study the amount of $\mathrm{Cd}$ found in Ompok pabda, Barbodes sarana, Tenualosa ilisha, Puntius sarena, Stinging catfish exceeded the limit $(0.02 \mathrm{mg} / \mathrm{kg})$ set by the (EU 2006) but in most fishes the concentration was below detection limit. Cadmium can accumulate in the human body that may causes prostate cancer and breast cancer (Saha and Zaman 2012), kidney dysfunction, skeletal damage and reproductive deficiencies in human (Commission of the European Communities 2001).

The concentration of $\mathrm{Pb}$ ranged between (BDL-6.85) $\mathrm{mg} / \mathrm{kg}$ that lower than the limit set by the EU is $0.3 \mathrm{mg} / \mathrm{kg}$ (EU 2006) except in Colisa lalia, Aila coila, Mastacembelus armatus, Glossogobius giuris, Nandus nandu, Barbodes sarana, Tenualosa ilisha, Labeo calbasu, Lepidocephalichthys guntea, Xenentodon cancila, Stinging catfish for 3 seasons. More or less similar result was found by Staniskiene et al. (2006); Copat et al. (2012). Lead is highly responsible for the reduced cognitive development and intellectual performance in children and increased blood pressure and cardio vascular disease in adults (Commission of the European Communities 2001).

FAO/WHO (1989) established limits for $\mathrm{Cu}$ in fish as 30.0 $\mathrm{mg} / \mathrm{kg}$ for human health. The value of $\mathrm{Cu}$ in the present study was varied between (0.03-32.44) $\mathrm{mg} / \mathrm{kg}$ far below the permissible limit except in Barbodes sarana exceeded the permissible limit. But $\mathrm{Cu}$ is necessary for the synthesis of haemoglobin but can cause harm at high concentrations (McCluggage 1991) such as liver and kidney damage (Agency for Toxic Substances and Disease Registry 2004).

$\mathrm{Ni}$ was found in very low concentration (BDL-0.986) $\mathrm{mg} / \mathrm{kg}$ in fish samples. The maximum concentration was set by (USFDA 1993) for $\mathrm{Ni}$ is $70-80 \mathrm{mg} / \mathrm{kg}$. The present concentration were far below the stipulated limit. Concentrations crossing the set limit may cause cancer of the lung and nasal cavity (USFDA 1993).

$\mathrm{Cr}$ is important element that helps the body use sugar, protein, and fat but excess amount have adverse effects on fish and wildlife (Akan et al. 2009) and sometimes $\mathrm{Cr}$ is carcinogenic (Institute of Medicine 2002). The concentration of $\mathrm{Cr}$ is below the limit (12-13) $\mathrm{mg} / \mathrm{kg}$ set by the United States Food and Drug Administration (USFDA 1993). Moreover, the deficiency of $\mathrm{Cr}$ can affect the glucose, lipid and protein metabolism and impaired growth (Akoto et al. 2014).

Fe concentration varied between (7.85-147.77) $\mathrm{mg} / \mathrm{kg}$ and highest concentration $147.77 \mathrm{mg} / \mathrm{kg}$ in Channa punctatus. Fe is essential element necessary for the production of hemoglobin, myoglobin and certain enzymes (Akoto et al. 2014). Fe deficiency causes anemia in human (Anderson and Fitzgerald 2010), weakness, inability to concentrate and susceptibility to infection (Akoto et al. 2014).

$\mathrm{Mn}$ in small amount needed for growth and prevention of cardiac arrest, heart attack, and stroke (Akoto et al. 2014; Ikem and Egiebor 2005). Acute toxicity causes psychologic and neurologic disorder (Saha and Zaman 2012). In present study the concentration of $\mathrm{Mn}$ in the fish muscles varied from $0.96-20.01 \mathrm{mg} / \mathrm{kg}$. Similar result found by (Akoto et al. 2014) and this result was lower than Begum et al. (2005) in Dhamondi Lake, Dhaka, Bangladesh (8.8-23.5 $\mu \mathrm{g} / \mathrm{g})$ and 0.59-11.74 $\mu \mathrm{g} / \mathrm{g}$ in Lake Tanganyika, Tanzania (Chale 2002).

Co concentrations was measured in all fish samples ranged from BDL-0.7 mg/kg wet weight. Co is beneficial to health but high level of Co may cause lung and heart effects and dermatitis (Agency for Toxic Substances and Disease Registry 2004).

The concentrations of $\mathrm{Al}$ ranged from $1.78-120.4 \mathrm{mg} / \mathrm{kg}$ in the fish muscles lower than the criteria for the maximum concentrations and criterion continuous concentration values of the US EPA (EPA 2002) water quality criteria. Aluminium frequently occur in the biosphere (Poleo 1995; GromyszKalkowska and Szubartowska 1999; Weng et al. 2002) and the in concentration of $\mathrm{Al}$ in rivers and lakes may cause fish death (Reitz et al. 1996; Alloway and Ayres 1997).

Ikem and Egiebor (2005) stated that some factors (the duration of exposure of fish to contaminants in water, feeding habit of fish, concentrations of contaminants in water column, water chemistry, contamination of fish during handling and processing) influenced the contamination of fish.

\section{Conclusion}

The findings of the present study indicate the fish community that are consumed regularly by the local people is being contaminated by different heavy metals discharged 
from the various industrial, urban as well as the agricultural activities occurred in the vicinity of the Meghna River. The elevated level of heavy metals found in most edible fishes ultimately will harm the human health. Therefore, the present research recommends that the point sources of heavy metals in the vicinity of the Meghna River should be strictly monitored for protecting the health of riverine ecosystem along with fish community.

\section{Acknowledgements}

The authors are grateful to the Bangladesh Council of Scientific and Industrial Research (BCSIR), Chittagong. The proposed research is a major contribution of Biodiversity, Environment and Climate Change Research Laboratory, Institute of Marine Sciences and Fisheries, University of Chittagong.

\section{References}

[1] Abdullah, M. R., Mohd Kamar, K. A., Mohd Nawi, M. N., Tarmizi Haron, A., and Arif, M. et al. (2009). "Industrialized building system: A definition and concept." Proc. of ARCOM Conference 2009, Notingham, UK.

[2] Abua Ikem and Nosa O. Egiebor, (2005). Assessment of trace elements in canned fishes (mackerel, tuna, salmon, sardines and herrings) marketed in Georgia and Alabama (United States of America), Journal of Food Composition and Analysis 18 (2005) 771-787. www.elsevier.com/locate/jfca

[3] Agency for Toxic Substances and Disease Registry, (2004). Agency for Toxic Substances and Disease Registry, Division of Toxicology, Clifton Road, NE, Atlanta, GA, available at: http://www.atsdr.cdc.gov/toxprofiles/.

[4] Ahmed, M. K., Baki, M. A., Islam, M. S., Kundu, G. K., Sarkar, S. K., Hossain, M. M. et al. (2015a). Human health risk assessment of heavy metals in tropical fish and shell fish collected from the river Buriganga, Bangladesh. Environ. Sci. Pollut. Res., http:// dx.doi.org/10.1007/s11356-015-4813-z.

[5] Ahmed, M. K., Shaheen, N., Islam, M. S., Al-Mamun, M. H., Islam, S., Banu, C. P. et al. (2015b). Trace elements in two staple cereals (rice and wheat) and associated health risk implications in Bangladesh. Environ. Monit. Assess. 187, 326-336.

[6] Ahmed, M. K., Shaheen, N., Islam, M. S., Al-Mamun, M. H., Islam, S., Mohiduzzaman, M., Bhattacharjee, L. et al. (2015c). Dietary intake of trace elements from highly consumed cultured fish (Labeorohita, Pangasius pangasius and Oreochromis mossambicus) and human health risk implications in Bangladesh. Chemosphere 128, 284-292.

[7] Akoto, O, Bismark Eshun, F, Darko, G. and Adei, E. et al. (2014). Concentrations and Health Risk Assessments of Heavy Metals in Fish from the Fosu Lagoon, Int. J. Environ. Res., 8(2):403-410, Spring 201

[8] Akan, J. C., Abdul -Rahman, F. I., Sodipo, O. A. and Akandu, P. I. et al. (2009). Bioaccumulation of some heavy metals of six fresh water fishes caught from Lake Chad in Doron Buhari, Borno State, Nigeria. J. Appl. Sci. in Environ. Sanit, 4 (2), 103-114.
[9] Akif, M., Khan, A. R., Sok, K., Min, K. S., Hussain, Z. and Maal-Abrar, M. et al. (2002). Textile effluents and their contribution towards aquatic pollution in the Kabul River (Pakistan). Journal of Chemical Society of Pakistan 24(2): 106-111.

[10] Alhashemi AH, Sekhavatjou MS, Kiabi BH. et al. (2012). Bioaccumulation of trace elements in water, sediment, and six fish species from a freshwater wetland, Iran. Microchem J 104:1-6.

[11] Alloway, B. J., Ayres, D. C. et al. (1997). Chemical Principles of Environmental Pollution. Second Edition. Blackie Academic \& Professional, London SEI 8HN, UK.

[12] Anawar HM, Akai J, Mostofa KM. et al. (2002). Arsenic poisoning in groundwater: Health risk and geochemical sources in Bangladesh. Environ Internat 27: 597-4.

[13] Bai, J., Xiao, R., Cui, B., Zhang, K., Wang, Q., Liu, X., Gao, H., Huang, L. et al. (2011). Assessment of heavy metal pollution in wetland soils from the young and old reclaimed regions in the Pearl River Estuary, South China. Environ. Pollut. 159, 817-824.

[14] Banerjee N, Nandy S, Kearns JK. et al. (2011). Polymorphisms in the TNF- $\alpha$ and IL10 gene promoters and risk of arsenic-induced skin lesions and other nondermatological health effects. Toxicol Sci 121(1): 132-39

[15] Begum, A., Amin, M. N., Kaneco, S. and Ohta, K. et al. (2005). Selected elemental composition of fish, Tilapia nilotica, Cirrhina mrigala and Clarius batrachus from the fresh water Dhanmondi Lake in Bangladesh. Food Chemistry, 93, 439-443.

[16] Camusso, M., Vigano, L., Baitstrini, R. et al. (1995). Bioaccumulation of trace metals in rainbow trout. Ecotox. Environ. Safe. 31: 133-141.

[17] Copat, C., Bella, F., Castaing, M., Fallico, R., Sciacca, S. and Ferrante, M. et al. (2012). Heavy Metals Concentrations in Fish from Sicily (Mediterranean Sea) and Evaluation of Possible Health Risks to Consumers. Bull. Environ. Contam. Toxicol., 88, 78-83.

[18] Commission of the European Communities, (2001). Commission Regulation (EC) No. 221/2002 of 6 February 2002 amending regulation (EC) No. 466/2002 setting maximum levels for certain contaminants in foodstuffs. Official Journal of the European Communities, Brussels, 6 February 2002.

[19] Dreher T. (2003). Evaluation of graphical and multivariate methods for classification of water chemistry data, Hydrogeology Journal, 11, 605-606.

[20] European Union, (2006). Setting maximum levels for certain contaminants in foodstuffs, Commission Regulation (EC) No 1881/2006; OJ L 364, 20.12.2006, p. 5

[21] European Union, (2002). Heavy Metals in Wastes, European Commission on Environment. Available at http://ec.europa.eu/environment/waste/studies/pdf/heavymetal sreport.pdf

[22] Fang Y, Sun X, Yang W. et al. (2014). Concentrations and health risks of lead, cadmium, arsenic, and mercury in rice and edible mushrooms in China. Food Chem 147: 147-51. 
[23] FAO/WHO, (1989). Evaluation of certain food additives and the contaminants mercury, lead and cadmium. WHO Technical Report, Series No. 505.

[24] Fernandes, C., Fontaínhas-Fernandes, A., Cabral, D., Salgado, M. A. et al. (2008). Heavy metals in water, sediment and tissues of Liza saliens from Esmoriz-Paramos lagoon, Portugal. Environ. Monit. Assess. 136: 267-275.

[25] Forti E, Salovaara S, Cetin Y. et al. (2011). In vitro evaluation of the toxicity induced by nickel soluble and particulate forms in human airway epithelial cells. Toxicol in Vitro 25:454-61

[26] Grigoratos, T., Samara, C., Voutsa, D., Manoli, E., Kouras, A. et al. (2014). Chemical composition and mass closure of ambient coarse particles at traffic and urban background sites in Thessaloniki, Greece. Environ. Sci. Pollut. Res. 21, 77087722.

[27] Gromysz-Kalkowska, K., Szubartowska, E. (1999). Aluminium - its ecological role and toxicity for animals. Medycyna Weterynaryjna 55(4), 229-223

[28] G. Afthan; G. Cumont; H. P. Dypdahl; K. Gadd; G. N. Havre; K. Julshamn; K. Kåverud; B. Lind; J. Loimaranta; M. Merseburg; A. Olsson; S. Piepponen; B. Sundström; B.J. Uppstad; T. Waaler; L. Winnerstam. et al. (2000). Determination of Metals in Foods by Atomic Absorption Spectrometry after Dry Ashing: NMKL1 Collaborative Study. LARS JORHEM. National Food Administration, Chemistry Division 2, Box 622, S-751 26 Uppsala, Sweden. Collaborators: Journal of AOAC International Vol. 83, No. 5, 20001207.

[29] Institute of Medicine, (2002). Dietary Reference Intakes for Vitamin A, Vitamin K, Arsenic, Boron, Chromium, Copper, Iodine, Iron, Manganese, Molybdenum, Nickel, Silicon, Vanadium, and Zinc. Institute of Medicine of the National Academies, The National Academy Press, 2101 Constitution Avenue, NW, Washington, DC, p. 773.

[30] Irwandi, J. and Farida, O. (2009). Mineral and heavy metal contents of marine fin fish in Langkawi Island, Malaysia, International Food Research Journal 16: 105-112 (2009).

[31] Islam, M. S., Ahmed, M. K., Habibullah-Al-Mamun, M., Islam, K.N., Ibrahim, M., Masunaga, S. et al. (2014). Arsenic and lead in foods: a potential threat to human health in Bangladesh. Food Addit. Contam. Part A 31 (12), 1982-1992.

[32] Islam, M. S., Ahmed, M. K., Habibullah-Al-Mamun, M., Hoque, M. F. et al. (2015a). Preliminary assessment of heavy metal contamination in surface sediments from a river in Bangladesh. Environ. Earth Sci. 73, 1837-1848.

[33] Islam, M. S., Ahmed, M. K., Raknuzzaman, M., HabibullahAl-Mamun, M., Masunaga, S. et al. (2015b). Metal speciation in sediment and their bioaccumulation in fish species of three urban rivers in Bangladesh. Arch. Environ. Contam. Toxicol. 68, 92-106.

[34] Islam, M. S., Ahmed, M. K., Raknuzzaman, M., HabibullahAl-Mamun, M., Islam, M. K. et al. (2015c). Heavy metal pollution in surface water and sediment: a preliminary assessment of an urban river in a developing country. Ecol. Indic. 48, 282-291.

[35] Ismaniza Ismail, Idaliza Mat Saleh, (2012). Analysis of heavy metals in water and fish (Tilapia sp.) samples from Tasik Mutlara, Puchong. The Malaysian Journal of Analytical Sciences, Vol 16 No 3 (2012): 346-352.
[36] Jaillon, L. and Poon, C. S. (2009). "The evolution of prefabricated residential building systems in hong kong: A review of the public and the private sector." Automation in Construction, Vol. 18, No. 3, pp. 239-248.

[37] Jordao, C. P., Pereira, M. G., Bellato, C. R., Pereira, J. L. and Matos, A. T. et al. (2002). Assessment of water systems for contaminants from domestic and industrial sewages. Environmental Monitoring Assessment 79(1): 75-100.

[38] John Edward Cantle, (1982). Atomic Absorption Spectrometry. Elsevier Scientific publishing Company, New York, 1982, pp -159-160.

[39] Kamal J. Elnabris, Shareef K. Muzyed, Nizam M. El-Ashgar. et al. (2012). Heavy metal concentrations in some commercially important fishes and their contribution to heavy metals exposure in Palestinian people of Gaza Strip ( Palestine ), Journal of the Association of Arab Universities for Basic and Applied Sciences(2013) 13, 44-51. http://dx.doi.org/10.1016/j.jaubas.2012.06.0

[40] Khan, S., Cao, Q., Zheng, Y. M., Huang, Y. Z., Zhu, Y. G. et al. (2008). Health risks of heavy metals in contaminated soils and food crops irrigated with wastewater in Beijing, China. Environ. Pollut. 152, 686-692.

[41] Martin, J. A. R., Arana, C. D., Ramos-Miras, J. J., Gil, C., Boluda, R. et al. (2015). Impact of 70 years urban growth associated with heavy metal pollution. Environ. Pollut. 196, 156-163.

[42] McCluggage D. (1991). Heavy metal poisoning, NCS Magazine. The Bird Hospital, CO, USA. Available at www.cockatiels.org/articles/Diseases/metals.html

[43] Md Saiful Islam, Md Kawser Ahmed \& Md Habibullah-AlMamun. et al. (2014). Determination of Heavy Metals in Fish and Vegetables in Bangladesh and Health Implications, Human and Ecological Risk Assessment: An International Journal, DOI: 10.1080/10807039.2014.950172

[44] Namminga, H. N., Wilhm, J. (1976). Effects of high discharge and an oil refinery cleanup operation bon heavy metals in water and sediments in Skeleton Creek. Proceedings of the Oklahoma Academy of Science, 56: 133-138.

[45] NAS-NRC, (1982). National, Drinking Water and Health, Academy of Sciences-National Research Council National Academic Press, Washington D.C.

[46] Özmen, H., Külahçı, F., Çukurovalı, A., and Doğru, M. et al. (2004). Concentrations of heavy metal and radioactivity in surface water and sediment of Hazar Lake (Elazığ, Turkey). Chemosphere, 55: 401-408.

[47] Öztürk, M., Özözen, G., Minareci, O., and Minareci, E. et al. (2008). Determination of heavy metals in of fishes, water and sediment from the Demirköprü Dam Lake (Turkey). Journal of Applied Biological Sciences, 2(3): 99-104.

[48] Pan K and Wang WX. (2012). Trace metal contamination in estuarine and coastal environments in China. Sci Tot Environ 421: $3-16$.

[49] Papagiannis I, Kagaloub I, Leonardos J. et al. (2004). Copper and zinc in four freshwater fish species from Lake Pamvotis (Greece). Environ Internat 30: 357-62.

[50] Pote, J., Haller, L., Loizeau, J. L., Bravo, A. G., Sastre, V., and Wildi, W. et al. (2008). Effects of a sewage treatment plant outlet pipe extension on the distribution of contaminants in the sediments of the Bay of Vidy, Lake Geneva, Switzerland. Bioresource Technol., 99: 7122-7131. 
[51] Poleo, A. B. S. (1995). Aluminium polymerization -- a mechanism of acute toxicity of aqueous aluminium to fish. Aquatic Toxicology 31(4), 347-356.

[52] Praveena, S. M., Radojevic, M., Abdullah, M. H., Aris, A. Z. et al. (2008). Application of sediment quality guidelines in the assessment of mangrove surface sediment in Mengkabong lagoon, Sabah, Malaysia. Iran. J. Environ. Health. Sci. Eng., 5 (1): $35-42$.

[53] Quddus, M. M. A., M. N., Sarker, A. K., Banerjee, et al. (1988). Studies of the Chondrichthyes Fauna (Sharks, Skates and Rays) of the Bay of Bengal. The Journal of NOAMI. Vol. 5, No. 1 \& 2. Pp. 19-39.

[54] Quddus, M. M. A. and Shafi, M. (1983). Bangapa sagarer Matsya Sampad (The Fisheries Resources of the Bay of Bengal). Kabir Publications, 38/3, Bangla Bazar, Dhaka, Bangladesh. $535 \mathrm{p}$.

[55] Rahman MM, Asaduzzaman M, and Naidu R. et al. (2013). Consumption of arsenic and other elements from vegetables and drinking water from an arsenic-contaminated area of Bangladesh. J Hazard Mater 262:1056-63.

[56] Rahman, A. K. A., Kabir, S. M. H., Ahmad, M., Ahmed, A. T. A., Ahmed, Z. U., Begum, Z. N. T., Hassan, M. A. and Khondker, M. et al. (2009). Encyclopedia of Flora and Fauna of Bangladesh, Vol. 24. Marine Fishes. Asiatic Society of Bangladesh, Dhaka. pp. 2-57.

[57] Rashed, M. N. (2001). Monitoring of environmental heavy metals in fish from Nasser Lake. Environ. Int., 27: 27-33.

[58] Rainbow, P. S., Amiard-Triquet, C., Amiard, J. C., Smith, B. D., Langston, W. J. et al. (2000). Observations on the interaction of zinc and cadmium uptake rates in crustaceans (amphipods and crabs) from coastal sites in UK and France differentially enriched with trace metals. Aquatic Toxicology 50,189 e 204 .

[59] Reitz, B., Heydorn, K., Pritzl, G. et al. (1996). Determination of aluminium in fish tissues bymeans of INAA and ICP-MS. Journal of Radioanalytical and Nuclear Chemistry216 (1), 113-116.

[60] Richard, R. B. (2005). "Industrialized building systems: Reproduction before automation and robotics." Automation in Construction, Vol. 14, No. 4, pp. 442-451.

[61] Roy, B. J., M. P. Dey, M. F. Alam and N. K. Singha, et al. (2007). Present Status of shark fishing in the marine water ofBangladesh.UNEP/CMS/MS/Inf/10.17p.http://www.cms.int/ bodies/meetings/regional/sharks/pdf_docs/Inf_10_Bangladesh Presentation_on_Shark_Fishing.pdff: Accessed 15 March, $\overline{2} 009$.

[62] Sanchez-Chardi, A., Lopez-Fuster, M. J., Nadal, J. et al. (2007). Bioaccumulation of lead, mercury, and cadmium in the greater white-toothed shrew, Crocidura russula, from the Ebro Delta (NE Spain): Sex- and age-dependent variation. Environ. Pollut. 145, 7-14.

[63] Saha, N and Zaman, M. R. (2012). Evaluation of possible health risks of heavy metals by consumption of foodstuffs available in the central market of Rajshahi City, Bangladesh; Environ. Monit. Assess. 185, 38673878.

[64] Sankar TV, Zynudheen AA, Anandan R. et al. (2006). Distribution of organochlorine pesticides and heavy metal residues in fish and shellfish from Calicut region, Kerala, India. Chemosphere 65: 583-90.

[65] Sekabira, K., Oryem Origa, H., Basamba, T. A., Mutumba, G., Kakudidi, E. et al. (2010). Assessment of heavy metal pollution in the urban stream sediments and its tributaries. Int. J. Environ. Sci. Technol. 7 (4), 435-446.

[66] Sharma RK, Agrawal M, and Marshall FM. et al. (2007). Heavy metals contamination of soil and vegetables in suburban areas of Varanasi, India. Ecotoxicol Environ Saf 66: $258-66$.

[67] Shuhaimi-Othmana, M., Pascoe, D. (2007). Bioconcentration and depuration of copper, cadmium, and zinc mixtures by the freshwater amphipod Hyalella azteca. Ecotoxicology and Environmental Safety 66 (1), $29 \mathrm{e} 35$.

[68] Sun, T. H., Zhou, Q. X., Li, P. J. et al. (2001). Pollution Ecology. Science Press, Beijing, China, pp. 160 194.

[69] Staniskiene, B., Matusevicius, P., Budreckiene, R. and Skibniewska, K. A. et al. (2006). Distribution of Heavy Metals in Tissues of Freshwater Fish in Lithuania; Polish J. of Environ. Stud., 15, 585-591.

[70] Tarra-Wahlberg, N. H., Flachierm A., Lane, S. N. and Sangfors, O. et al. (2001). Environmental impacts and metal exposure of aquatic ecosystems in rivers contaminated by small scale gold mining: The Puyango River Basin, Sourthen Ecuador. Science of the Total Environment 278 (1-3): 239261.

[71] Thanoon, W. A., Wah Peng, L., Abdul Kadir M. R., Jaafar, M. S., and Salit, M. S. et al. (2003). "The essential characteristics of industrialised building system." Proc. of International Conference on Industrialized Building Systems, Kualalumpur, Malaysia, pp. 283-292.

[72] Tuzen M. (2009). Toxic and essential trace elemental contents in fish species from the Black Sea, Turkey. Food Chem Toxicol 47, 1785-90.

[73] USFDA, (1993). United States Food and drug administration, Guidance document for chromium in shellfish. DHHS/PHS/FDA/CFSAN/Office of seafood, Washington D. C.

[74] Vieira C, Morais S, Ramos S. et al. (2011). Mercury, cadmium, lead and arsenic levels in three pelagic fish species from the Atlantic Ocean: Intra- and inter-specific variability and human health risks for consumption. Food Chem Toxicol 49(4): 923-32.

[75] Weng, L., Temminghoff, E. J. M., Van Riemsdijk, W. et al. (2002). Aluminium speciation innatural waters: measurement using Donnan membrane technique and modellingusing NICA-Donnan. Water Research 36(17), 4215-4226.

[76] Wilson, B., Pyatt, F.B. (2007). Heavy metal dispersion persistence, and bioaccumulation around an ancient copper mine situated in Anglesey, UK. Ecotoxicol. Environ. Saf. 66, 224-231.

[77] WHO (World Health Organization), (1995). Lead. Geneva: Environmental Health Criteria, Geneva, Switzerland.

[78] WHO (World Health Organisation), (1972). Evaluation of certain food additives and the contaminants mercury, lead, cadmium. 16 th Report of the Joint FAO/WHO Expert Committee on Food Additives. Technical Report Series 505, Geneva. 
[79] WHO (World Health Organisation), (1987). Evaluation of certain food additives and contaminants. 33rd Report of the joint FAO/WHO Expert Committee on Food Additives. WHO Technical Report Series 776, Geneva, 80 pp.

[80] Yi, Y., Yang, Z., Zhang, S. et al. (2011). Ecological risk assessment of heavy metals in sediment and human health risk assessment of heavy metals in fishes in the middle and lower reaches of the Yangtze river basin. Environ. Pollut. 159, 2575-2585.
[81] Zhang, C., Qiao, Q., Piper, J. D. A., Huang, B. et al. (2011). Assessment of heavy metal pollution from a Fe-smelting plant in urban river sediments using environmental magnetic and geochemical methods. Environ. Pollut. 159, 3057-3070.

[82] Zhou, Q. X., Kong, F. X., Zhu, L. et al. (2004). Ecotoxicology: Principles and Methods. Science Press, Beijing, pp. 161-217.

[83] Zhou, Q. X. (1995). Ecology of Combined Pollution. China Environmental Science Press, Beijing, pp. 1-29. 